\title{
Spacing distributions for rhombus billiards
}

\author{
Benoît Grémaud ${ }^{\dagger}$ and Sudhir R. Jain ${ }^{\dagger \dagger}$ \\ † Laboratoire Kastler Brossel, Université Pierre et Marie Curie, T12, E1 \\ 4, place Jussieu, 75252 Paris Cedex 05, France \\ ${ }^{\dagger \dagger}$ Theoretical Physics Division, Bhabha Atomic Research Centre, \\ Central Complex, Trombay, Mumbai 400 085, India
}

(November 15, 2018)

\begin{abstract}
We show that the spacing distributions of rational rhombus billiards fall in a family of universality classes distinctly different from the Wigner-Dyson family of random matrix theory and the Poisson distribution. Some of the distributions find explanation in a recent work of Bogomolny, Gerland, and Schmit. For the irrational billiards, despite ergodicity, we get the same distribution for the examples considered - once again, distinct from the Wigner-Dyson distributions. All the results are obtained numerically by a method that allows us to reach very high energies.
\end{abstract}

Statistical analysis of level correlations of a quantum system is one of the many ways to study the effects of chaotic behaviour of its classical counterpart [1]. For such complex systems, the fluctuations are very well described by the random matrix theory, giving rise to three classes of universality corresponding to orthogonal, unitary and symplectic ensembles (OE, UE and SE). On the other hand, for integrable systems, the short range correlations follow the Poisson distribution. Rhombus billiards [2] are peculiar as they are pseudo-integrable systems and for this reason, their statistical properties belong to another class of universality [3]. These non-integrable systems are termed pseudointegrable as the dynamics occurs on a multiply-connected, compact surface in the phase space. For example, in the case of $\pi / 3$-rhombus billiard, the invariant integral surface is a sphere with two handles [2. 4 . It has been shown that the short-range properties (spacing distribution) can be fitted by Brody distributions [5] with parameters depending on the genus [6]. However, a very small number of levels were used to achieve the statistics and, as it was outlined by the authors, the parameters were smoothly changing with the number of levels considered. This last effect is probably a consequence of the pseudo-integrability and thus one has to consider levels lying very high in energy to have converged statistics. Furthermore, Brody distributions are not very convenient for two reasons: (i) they are not on a firm theoretical basis like random matrix theory and so, one can not gain too much knowledge about the system from the Brody parameter; (ii) their behaviour at small spacing is not linear, whereas it is so for rhombus billiards. On the contrary, in a recent paper [7], Bogomolny et al. have proposed a model derived from the Dyson's stochastic Coulomb gas one 88,9] : eigenvalues are considered as classical particles on a line, with a two-body interaction potential given by $V(x)=-\ln (x)$. Contrary to Dyson's model, where all possible pairs are considered, the same interaction is restricted only to nearest-neighbours. Hereafter, this model will be referred as short ranged Dyson's model (SRDM). The joint probability obtained gives rise to spacing distributions showing linear level repulsion and exponential decrease for large spacing. More precisely, the nearest-neighbour (NN) and next-nearest-neighbour (NNN) distributions are :

$$
P(s)=4 s e^{-2 s} \quad \text { and } \quad P_{2}(s)=\frac{8}{3} s^{3} e^{-2 s}
$$

It is worth noting that exactly the same functional form was used in the past 10 to explain the intermediate spacing distribution for a rectangle billiard with a flux line - an Aharanov-Bohm billiard. In this recent work [7], it is also shown that the level statistics of some rhombus billiards agree very well with these distributions. However, only rhombi with rational angles and with Dirichlet boundary conditions on both $x$ and $y$ axis (i.e. right-angled triangle) were studied. In this Letter, we extend the preceding study to rational billiards with Neumann boundary conditions (i.e. "pure" rhombus) and also to irrational billiards (both classes of boundary conditions). Of course, in a rhombus, making the shorter (longer) diagonal Neumann means that one is considering a larger obtuse (acute) triangle. So, the modifications are expected but here they are non-trivial.

The spectral properties of these systems which are non-integrable and yet non-chaotic is thus an important unsettled problem. The solution of this problem is partly in devising numerical techniques that allow one to go to higher energies, and, partly in developing statistical models like the SRDM (Ref. [7])mentioned above. In this Letter, we first discuss the method and then use the levels in high energy range to show agreements and disagreements with the results in [7. To give an idea, the efficiency of the method is such that we were able to compute a very large number of levels (up to 36000 for a given rhombus and a given symmetry class), so that the statistical properties are fully converged. In 
the later part of the Letter, we show the effects of both, the boundary conditions and the irrationality on the level spacing distributions.

The Schrödinger equation for a particle moving freely in a rhombus billiard (shown by Fig. 1) is simply :

$$
-\frac{\hbar^{2}}{2 m}\left(\frac{\partial^{2}}{\partial x^{2}}+\frac{\partial^{2}}{\partial y^{2}}\right) \psi(x, y)=E \psi(x, y)
$$

with the additional condition that $\psi(x, y)$ is vanishing on the boundary (Dirichlet conditions). The geometry of the system leads to a natural change of coordinates : the two new axes cross at the centre and are parallel to the edges of the billiard (see Fig. 11) :

$$
\left\{\begin{array}{l}
\mu=\frac{1}{2}\left(\frac{x}{\cos \theta}-\frac{y}{\sin \theta}\right) \\
\nu=\frac{1}{2}\left(\frac{x}{\cos \theta}+\frac{y}{\sin \theta}\right) .
\end{array}\right.
$$

In this new coordinate system, the original rhombus is mapped onto a square of length $L$ and thus, in this coordinate system, the boundary conditions separate, of course at the price of a slightly more complicated Schrödinger equation :

$$
-\frac{\hbar^{2}\left(\partial_{\mu \mu}^{2}+\partial_{\nu \nu}^{2}-2 \cos (2 \theta) \partial_{\mu \nu}^{2}\right)}{2 m \sin ^{2}(2 \theta)} \psi(\mu, \nu)=E \psi(\mu, \nu)
$$

The change $\mu \rightarrow \frac{2}{L} \mu, \nu \rightarrow \frac{2}{L} \nu$ and $E \rightarrow\left(\frac{2}{L}\right)^{2} \frac{m}{\hbar^{2}} E$ gives rise to the scaled Schrödinger equation (after multiplication by $\left.2 \sin ^{2}(2 \theta)\right)$ :

$$
-\left(\partial_{\mu \mu}^{2}+\partial_{\nu \nu}^{2}-2 \cos (2 \theta) \partial_{\mu \nu}^{2}\right) \psi=2 \sin ^{2}(2 \theta) E \psi
$$

the boundary condition being then at the points $\mu= \pm 1$ and $\nu= \pm 1$.

To solve the eigenvalue problem, a possible idea is to expand any wavefunction in a basis satisfying the boundary conditions:

$$
\psi(\mu, \nu)=\sum_{n_{\mu}, n_{\nu}=0}^{\infty} a\left(n_{\mu}, n_{\nu}\right) \phi_{n_{\mu}}(\mu) \phi_{n_{\nu}}(\nu)
$$

The simplest choice is the Fourier sine and cosine series. Unfortunately, the operator $\partial_{\mu \nu}^{2}$ has no selection rules in this basis, thus the matrix representation of the left part of the Schrödinger equation (5) is totally filled. Numerically, we will approximate the wavefunction by keeping only a (large) number of terms in the preceding serie. For this system and for many others like coulomb systems, it has been observed that the rate of convergence of the serie is much slower when the matrix is filled than when selection rules occur.

To avoid this difficulty, we introduce the following basis for each coordinate $\mu$ and $\nu$ :

$$
\phi_{n}(u)=\left(1-u^{2}\right) C_{n}^{\left(\frac{3}{2}\right)}(u)
$$

where $C_{n}^{\alpha}$ are Gegenbauer polynomials [11]. This basis is complete and all operators appearing in equation (5) have selection rules. More precisely, we have

$$
\left|\Delta n_{\mu}\right|,\left|\Delta n_{\nu}\right| \leq 2 \quad \Delta n_{\mu}+\Delta n_{\nu}=0, \pm 2, \pm 4
$$

Furthermore, all matrix elements are analytically known and are given by simple polynomial expressions of the two quantum numbers $\left(n_{\mu}, n_{\nu}\right)$. The only difficulty is the non-orthogonality of the basis, that is $\left\langle n^{\prime} \mid n\right\rangle$ does not reduce to $\delta_{n n^{\prime}}$, but also shows the preceding selection rules. 
This basis also allows us to take directly into account the symmetries of the original problem, namely the reflections with respect to the $x$-axis $\left(S_{x}\right)$ or the $y$-axis $\left(S_{y}\right)$. In $(\mu, \nu)$ coordinates, they become :

$$
S_{x}\left\{\begin{array}{l}
\mu \rightarrow \nu, \\
\nu \rightarrow \mu
\end{array} ; \quad S_{y} \quad\left\{\begin{array}{l}
\mu \rightarrow-\nu, \\
\nu \rightarrow-\mu .
\end{array}\right.\right.
$$

Using the properties of the Gegenbauer polynomials [11, we are able to construct four different bases in which the two operators $S_{x}$ and $S_{y}$ are simultaneously diagonal with eigenvalues, $\epsilon_{x}= \pm 1$ and $\epsilon_{y}= \pm 1$. Of course, this transformation preserves the selection rules and hence the band structure. We shall denote the eigenfunctions vanishing on both the diagonals by $(--)$ - and not vanishing on either by $(++)$-parity classes.

The original Schrödinger equation is thus transformed to a generalized eigenvalue problem :

$$
A|\psi\rangle=E B|\psi\rangle
$$

where $A$ and $B$ are real, sparse and banded matrices. This kind of system is easily solved using the Lanczos algorithm [12]. It is an iterative method, highly efficient to obtain few eigenvalues and eigenvectors of very large matrices. We obtain typically 100 eigenvalues of a $10000 \times 10000$ matrix in few minutes on a regular workstation. The results presented here have been obtained by diagonalizing matrices of size up to 203401 for a bandwidth equal to 903 . For such matrices, we obtain 200 eigenvalues in 10 minutes on a Cray C98. The number of levels $(\simeq 36000)$ that we are able to compute in this way is a bit larger than with usual boundary matching methods $(\simeq 20000)$, which are nevertheless restricted to rational angles. On the other hand, very recent methods developed by Vergini et al [13] seems to be more efficient (they were able to reach energy domain around the $142,000^{\text {th }}$ state for the stadium billiard).

For the present study, various values of angle have been used :

$$
\frac{3 \pi}{10}, \quad \frac{(\sqrt{5}-1) \pi}{4}, \quad \frac{\pi}{\pi}, \quad \frac{\pi}{3} \quad \frac{3 \pi}{8} \quad \text { and } \quad \frac{7 \pi}{18}
$$

for both $(++)$ and $(--)$ parity [14]. For all cases, only levels above the $10000^{\text {th }}$ one have been considered, to avoid peculiar effects in the statistics and at least 5000 levels (up to 24000) have been used for each case. The convergence of the statistics has been checked by systematically varying the energy around which levels were taken. This is shown in Fig. 2, where we have plotted the following quantity :

$$
\int_{0}^{\infty} d s\left(N_{0}(s)-N_{n}(s)\right)^{2}
$$

with respect to the number $n$, for $\frac{3 \pi}{10}$ (top) and $\frac{\pi}{3}$ (bottom) billiards $\left((++)\right.$ parity). $N_{0}(s)$ is the cumulative NN distribution obtained with the 5000 highest states, whereas $N_{n}(s)$ is the cumulative NN distribution obtained with levels $n$ to $n+4999$. One can thus clearly see that the statistics become energy independent (up to fluctuations) only for levels above the $10000^{\text {th }}$ state, which emphasizes the choice of keeping only those states.

In [7], it was shown that for the $\frac{3 \pi}{10}(--)$ billiard, both NN and NNN statistics were following the formula (1). We, of course, reproduce this result, as shown in Fig. 3(a). However, the same billiard, but with Neumann-Neumann boundary conditions does not follow the same distribution laws, but rather lies in between OE and SRDM distributions, as it is shown in Fig. 3(a). The deviations are obviously much larger than statistical fluctuations. The difference is emphasized by looking at the behavior of the NNN for small spacings (see Fig. $3($ b)). Indeed, whereas for the (--) symmetry, the observed power law is $s^{4}$ in the cumulative distribution (i.e., $s^{3}$ for $P(1, s)$ ), it is close to $s^{5}$ for the $(++)$ case (i.e. $s^{4}$ for $P(1, s)$ ), which is the OE prediction. This dependency of the statistics with respect to the boundary conditions has already been observed in other systems like 3D Anderson's model [15]. However the present results are more surprising as there are $\theta$ values for which there is practically no difference between the two symmetry classes. Indeed, Fig. 1 shows the NN (cumulative) distributions for $\frac{3 \pi}{8}$ and $\frac{7 \pi}{18}$. Besides the statistical fluctuations, one cannot distinguish between the two symmetry classes, whereas the distributions differ $: \frac{3 \pi}{8}$ is well described by SRDM, whereas $\frac{7 \pi}{18}$ lies between OE and SRDM.

The case of the $\frac{\pi}{3}$ billiard is the most peculiar, since the $(--)$ parity is integrable whereas the $(++)$ spacing distributions agree with SRDM.

All the rhombi considered are not ergodic, as their genus are finite (e.g., two for the $\pi / 3$-rhombus). In contrast, for irrational angle, "the genus is infinite", and so one could expect a rather different behaviour. Although the concept 
of genus is applicable only to compact surfaces, we have stated the above phrase in quotes in the following sense. As an irrational rhombus is approximated via continued fraction expansion, the larger and larger denominators will appear, implying larger genus surfaces, and eventually "infinite". It is quite possible, and it may, in fact, be true, that this limit is singular. As a result, from the rational convergents, it may not be possible to say something about the irrational billiard.

Fig. 5 displays NN and NNN statistics for $\frac{\pi}{\pi}$ and $\frac{(\sqrt{5}-1) \pi}{4}$ (both symmetry classes) billiards. NN distributions are on top of each other, which is interesting if one believes that the genus is the relevant parameter. On the other hand, from the ergodicity, one could expect the distributions to be OE, which is not the case, even if the small spacing behavior of NNN statistics seems to show the same power law $s^{5}$ (for cumulative). Thus, if Ref. [7] seems to give one class of universality, there must be other classes of universality lying between SRDM and OE, especially for irrational angles. The other possibility is that, although numerically stationary in a wide range of energy, the spacing distributions of irrational rhombus may evolve exceptionally slowly to OE. If it is the case, one will probably have to find the final answer in a much higher energy range, for which other numerical methods will have to be used 13 .

The present study also raises the question of the semiclassical understanding of the boundary dependence of the distributions. Due to change in boundary conditions, actions, Maslov indices and also the edge orbits will change resulting in a difference, but the whole explanation of this boundary dependence probably lies beyond these simple considerations. Spectral fluctuations in some of the pseudo-integrable billiards have been studied in detail using the periodic orbit theory. From the detailed information about the periodic orbits 仼, it was shown that the spectral rigidity is non-universal [3] with a universal trend. We hope that the method presented here, and the ensuing numerical results will help us to model the spectral fluctuations of these apparently simple non-integrable quantum systems.

To summarize, we have casted the problem of a particle in rhombus-shaped enclosure in a way that allows us to go to very high energies. This has led us to confidently obtain statistical results on spacing distributions which are well converged. Subsequently, we have shown that for some rational billiards, the fluctuations agree well with the results recently obtained [7]. However, we have given several examples where the recent model does not explain the obtained distributions. It has been shown that boundary conditions play an important role. Finally, for the irrational rhombus billiards, the distributions seem to be identical for the examples considered. Significantly though, the distribution is still not in the Wigner-Dyson family. We believe that these results point in the direction of having a family of universality classes which, in essence, leads to nonuniversality with a universal trend for pseudointegrable billiards.

We acknowledge stimulating discussions with D. Delande and E. Bogomolny. CPU time on a Cray C98 computer has been provided by IDRIS. Laboratoire Kastler Brossel is laboratoire de l'Université Pierre et Marie Curie et de l'Ecole Normale Supérieure, unité associée 18 du CNRS.

[1] O. Bohigas, M.-J. Giannoni, and C. Schmit, Phys. Rev. Lett. 52, 1 (1984).

[2] D. Biswas and S. R. Jain, Phys. Rev. A 42, 3170 (1990); B. Eckhardt, J. Ford, and F. Vivaldi, Physica D 13, 339 (1984); for a review, see S. R. Jain and S. V. Lawande, Proc. Indian Natl. Sci. Acad. A 61, 275 (1995).

[3] H. D. Parab and S. R. Jain, J. Phys. A 29, 3903 (1996).

[4] S. R. Jain and H. D. Parab, J. Phys. A 25, 6669 (1992).

[5] T.A. Brody, Lett. Nuovo Cimento 7, 482 (1973).

[6] A. Shudo and Y. Shimizu, Phys. Rev. E 47, 54 (1993).

[7] E.B. Bogomolny, U. Gerland and C. Schmit, submitted to Phys. Rev. Lett.

[8] M.L. Mehta, Random matrices, Academic Press (1991)

[9] F. Haake, Quantum signatures of Chaos, Springer-Verlag (1992)

[10] G. Date, S. R. Jain, and M. V. N. Murthy, Phys. Rev. E 51, 198 (1995).

[11] M. Abramowitz and A. Segun, Handbook of Mathematical Functions, Dover Publications (1968)

[12] T. Ericsson and A. Ruhe, Math. Comp. 35, 1251 (1980) and references therein.

[13] E. Vergini and M. Saraceno, Phys. Rev. E 52, 2204 (1995)

[14] The (--) parity for the $\frac{\pi}{3}$ rhombus is not shown as it is integrable.

[15] D. Braun, G. Montambaux and M. Pascaud, submitted to Phys. Rev. Lett. 


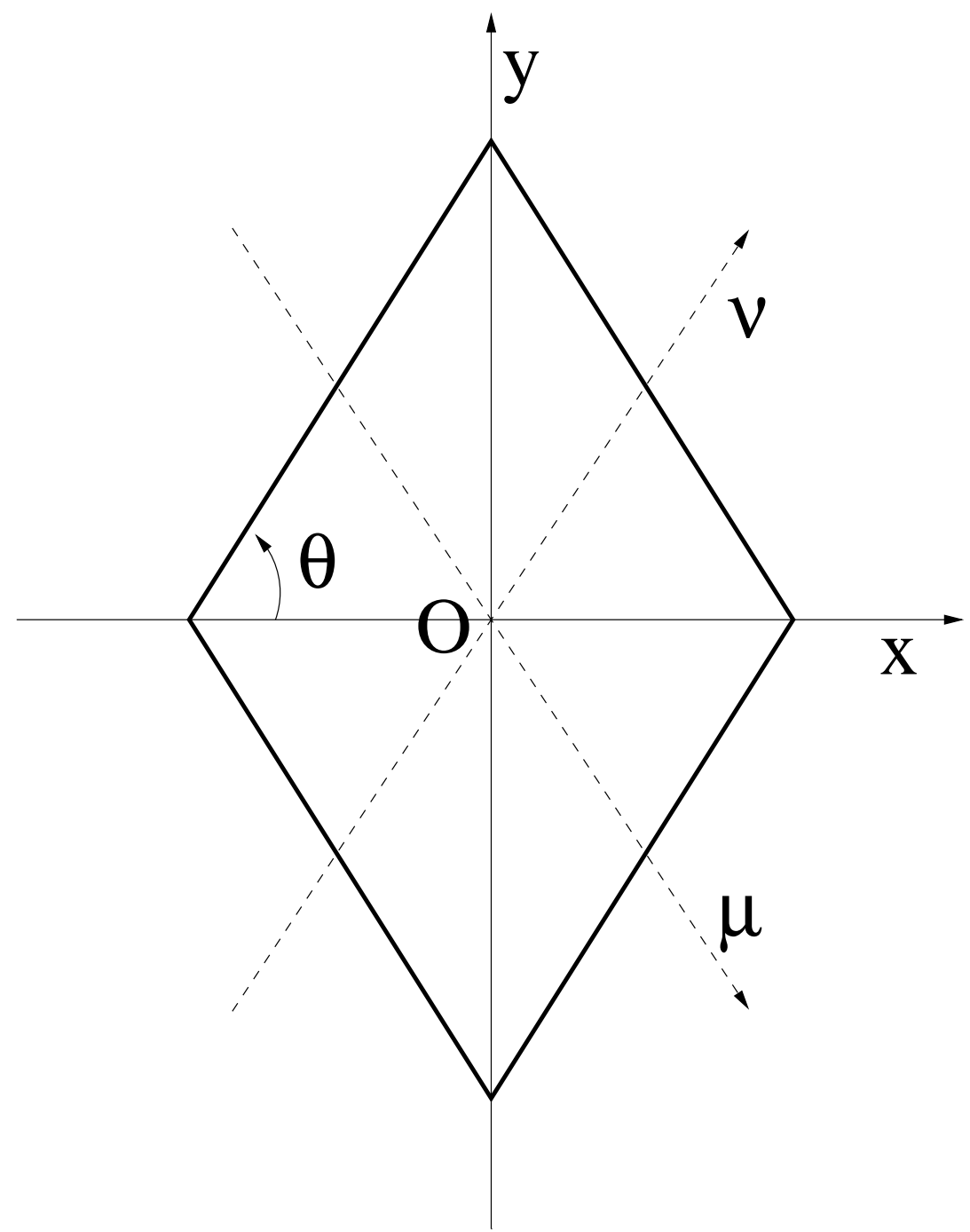

FIG. 1. Rhombus-shaped enclosure in which the particle moves freely with elastic bounces on the boundary, the quantum problem corresponds to imposing the Dirichlet conditions for the wavefunctions. The system being symmetric under reflections with respect to the $x$-axis or the $y$-axis, Dirichlet or Neumann boundary conditions can be imposed on the both axis, leading to four different classes of symmetry. By considering axes crossing at the centre $O$ of the system and parallel to the edges of the billiard, a non-orthogonal coordinate system $(\mu, \nu)$ is constructed in which the Dirichlet boundary conditions on the enclosure separate (see text). 

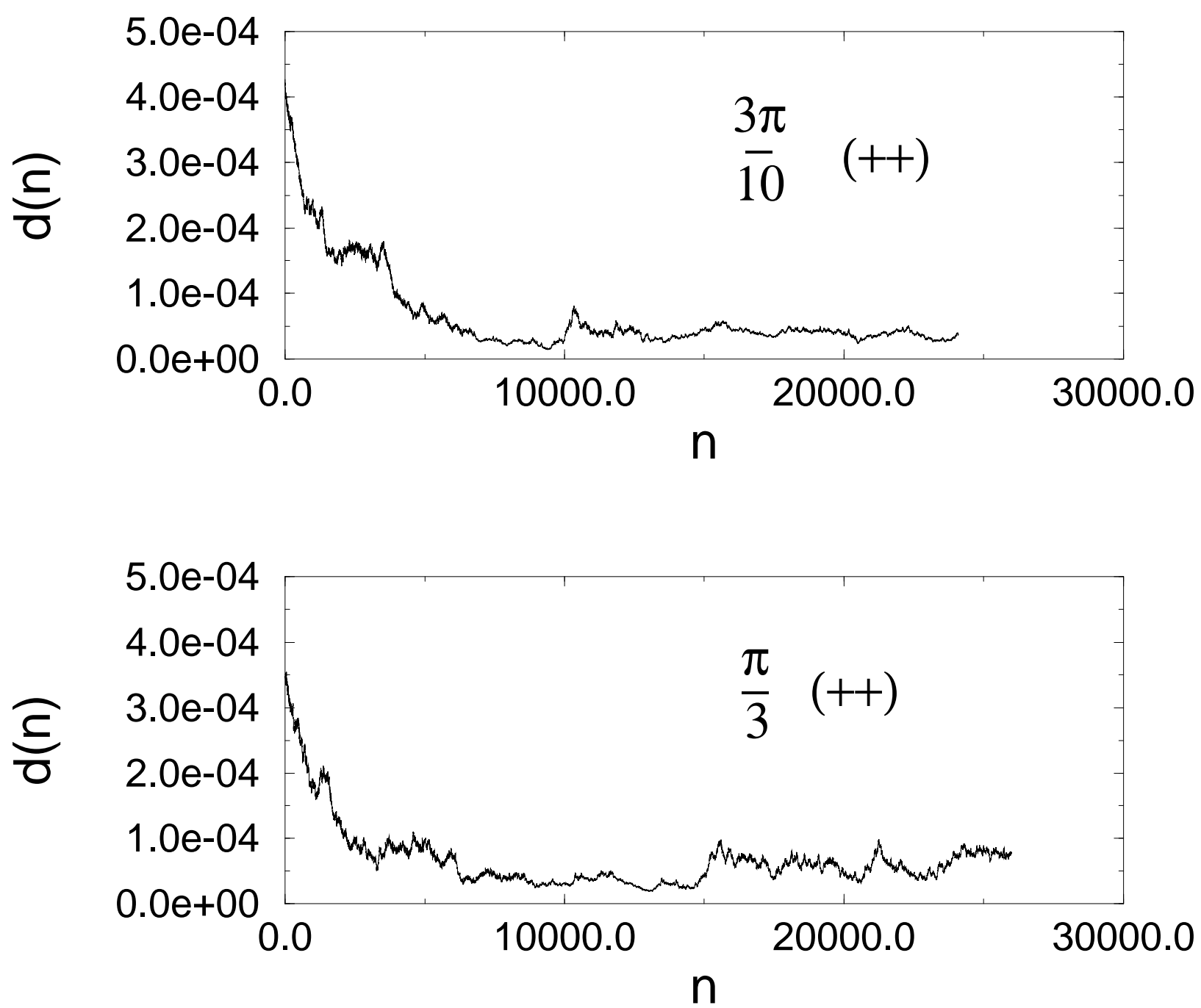

FIG. 2. "Difference" (see equation (12)) between the NN statistics obtained with the 5000 highest states and the NN statistics obtained with levels $n$ to $n+4999$, as a function of $n$, for both $\frac{3 \pi}{10}$ (top) and $\frac{\pi}{3}$ (bottom) (++ parity). Above the $10000^{\text {th }}$ level, the distributions become energy independent (apart fluctuations). 

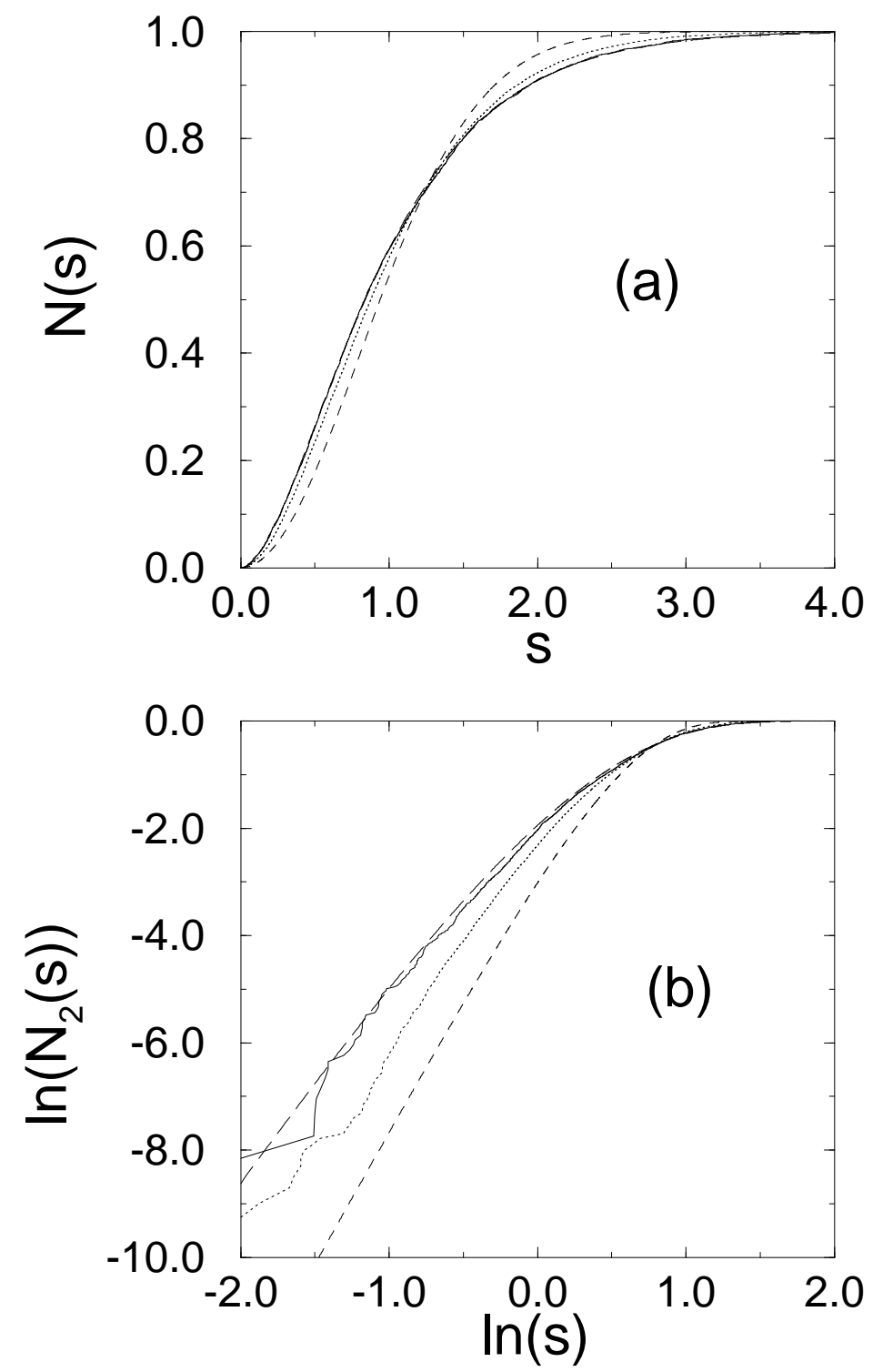

FIG. 3. On the top is shown the cumulative distribution of nearest neighbour spacings for the $\frac{3 \pi}{10}$ rhombus. The dotted line corresponds to Neumann-Neumann $(++)$ boundary conditions on both $x$ - and $y$-axis, the continuous line corresponding to Dirichlet-Dirichlet (--) boundary conditions. The two distributions are clearly different, the deviation being larger than statistical fluctuations. The $(--)$ symmetry class is exactly on the top of the distribution introduced by Bogomolny et al. (SRDM) given by equation (1), corresponding to the long-dashed line. The $(++)$ distribution lies in between SRDM and OE prediction (given by the dashed line). This difference is emphasized in figure (b) depicting the next-nearest neighbour distributions (cumulative) for the same billiards (ln $-\ln$ plot). Again, the (--) (continuous line) symmetry class is exactly on the top of SRDM (long dashed line), whereas the $(++)$ symmetry class (dotted line) lies in between SRDM and OE (dashed line). Especially the behaviours for small spacing are very different : $(--)$ shows a $s^{4}$ power law, whereas it is $s^{5}$ for $(++)$, the OE prediction. 


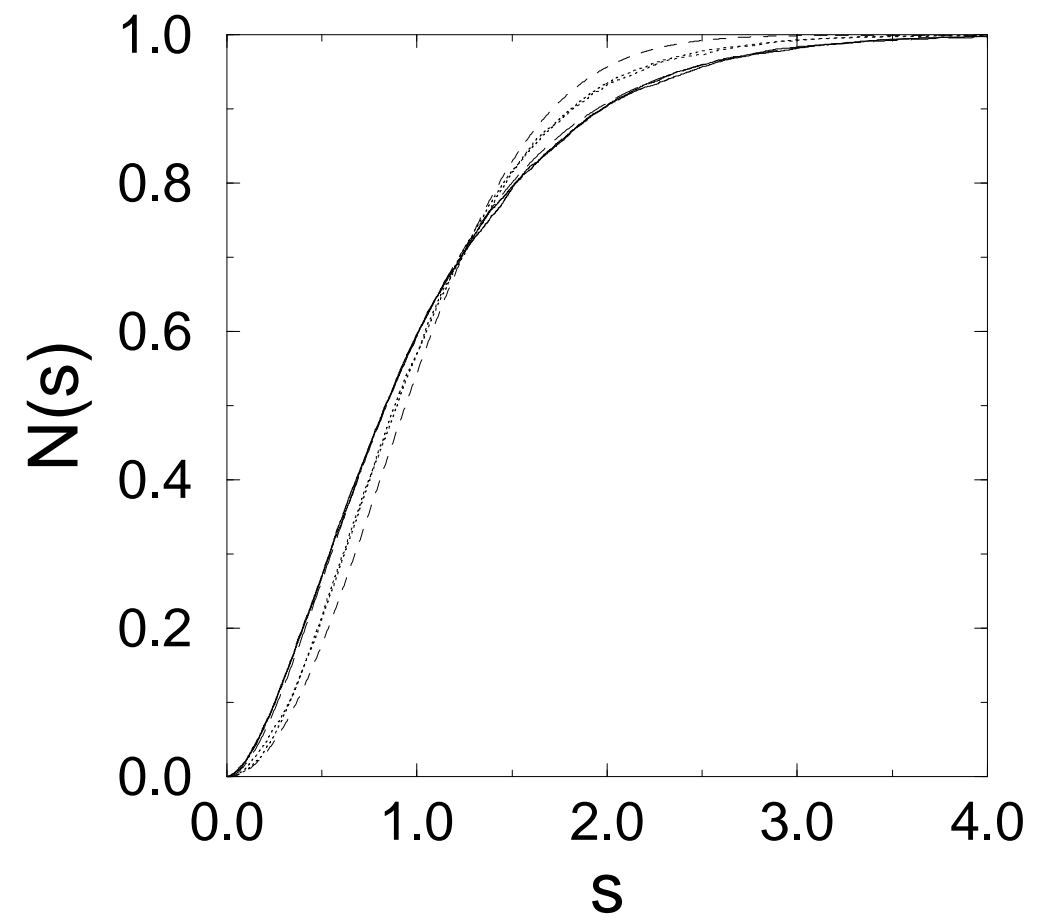

FIG. 4. Spacing distributions (cumulative) for two rational billiards : $\frac{3 \pi}{8}$ (continuous lines) and $\frac{7 \pi}{18}$ (dotted lines), for both $(++)$ and $(--)$ symmetry classes. Contrary to the $\frac{3 \pi}{10}$ billiard (see Fig. 3), there is no difference between the two symmetry classes : for each billiard the two curves lie on top of each other. Furthermore, these two billiards show distinct spacing distributions, the $\frac{3 \pi}{8}$ one corresponds exactly to SRDM (long dashed line) whereas the $\frac{7 \pi}{18}$ one is much closer to OE prediction (dashed line). 

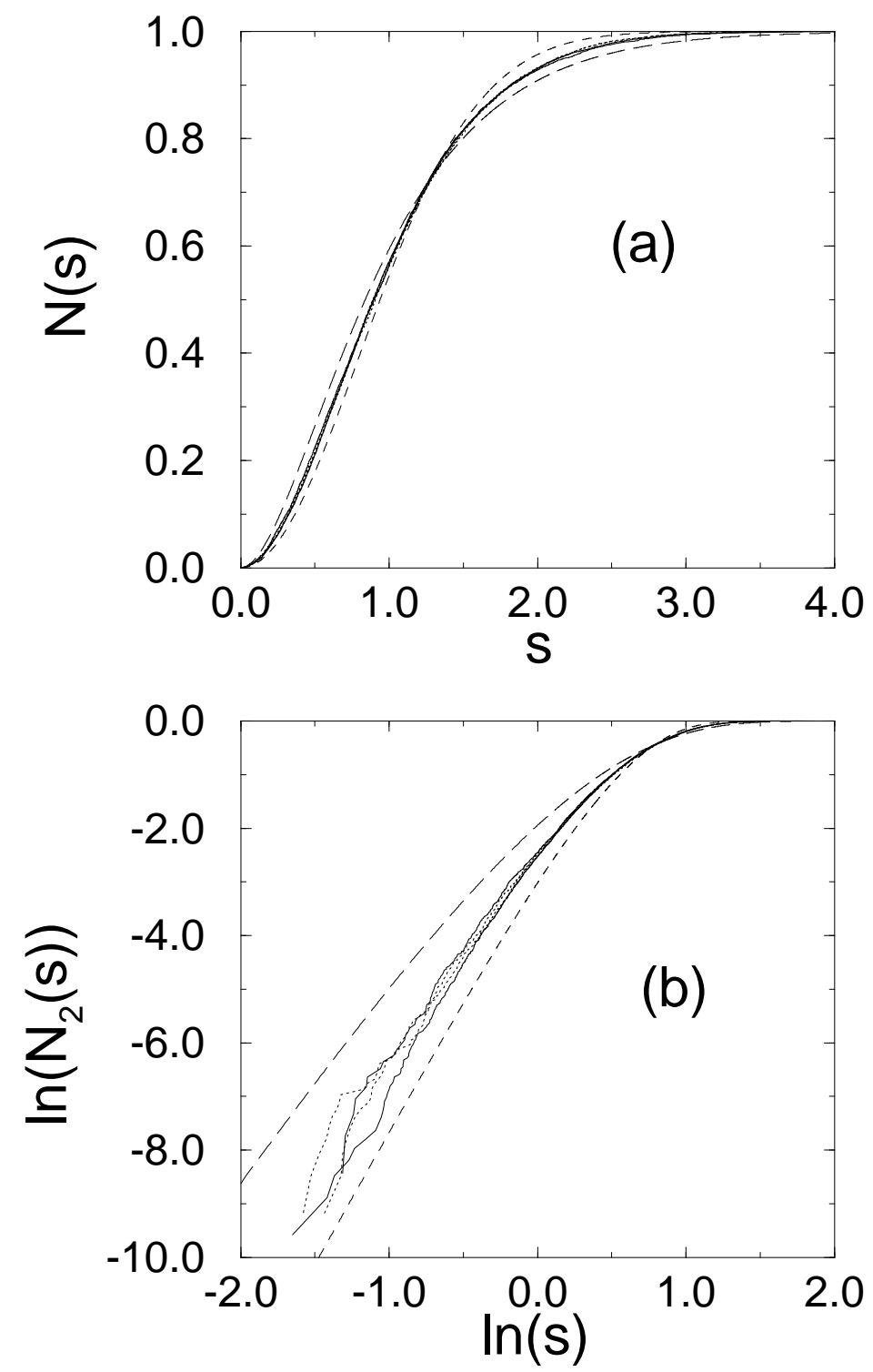

FIG. 5. Nearest neighbour (a) and next-nearest neighbour (b) distributions for two irrational billiards : $\frac{\pi}{\pi}$ (continuous lines) and $\frac{(\sqrt{5}-1) \pi}{4}$ (dotted lines) for both $(++)$ and $(--)$ symmetry classes. Contrary to the rational billiards, the genus of these billiard is "infinite" (see text for explanation) and so the classical dynamics is ergodic. The fact that all the four distributions lie on top of each other is quite remarkable and may be related to the fact that these billiards have the "same" genus. However, from the ergodicity one could expect the distributions to be OE-like, which is not the case. They rather lie between SRDM (long dashed) and OE (short dashed). Still, the small spacing behaviour of the next-nearest neighbour distributions shows a $s^{5}$ power law, i.e. corresponding to OE. 